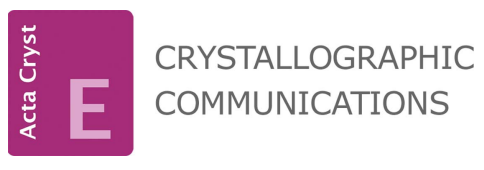

ISSN 2056-9890

Received 12 December 2016

Accepted 9 February 2017

Edited by H. Ishida, Okayama University, Japan

Keywords: crystal structure; tetrahedral tetrachloridocuprate ion; tetramethylammonium ion.

CCDC reference: 1531866

Supporting information: this article has supporting information at journals.iucr.org/e

\section{Crystal structure of the monoclinic phase (phase IV) of bis(tetramethylammonium) tetrachlorido- cuprate(II)}

\author{
Gorgui Awa Seck, ${ }^{a}$ Libasse Diop ${ }^{\mathrm{a} *}$ and Allen G. Oliver ${ }^{\mathrm{b}}$
}

\begin{abstract}
a'Laboratoire de Chimie Minérale et Analytique, Département de Chimie, Faculté des Sciences et Techniques, Université Cheikh Anta Diop, Dakar, Senegal, and ${ }^{\mathbf{b}}$ Department of Chemistry and Biochemistry, University of Notre Dame, IN 46557-5670, USA. *Correspondence e-mail: dlibasse@gmail.com
\end{abstract}

The crystal structure of the low-temperature monoclinic phase of the title compound, $\left[\left(\mathrm{CH}_{3}\right)_{4} \mathrm{~N}\right]_{2}\left[\mathrm{CuCl}_{4}\right]$, was determined at $120 \mathrm{~K}$. The structure of the room-temperature phase has been determined in the orthorhombic space group Pmcm [Morosin \& Lingafelter (1961). J. Phys. Chem. 50-51; Clay et al. (1975). Acta Cryst. B31 289-290]. The asymmetric unit consists of one discrete tetrachloridocuprate anion with a distorted tetrahedral geometry and two tetramethylammonium cations. In the crystal, the cations and the anions are linked via weak $\mathrm{C}-\mathrm{H} \cdots \mathrm{Cl}$ hydrogen bonds.

\section{Chemical context}

The title compound undergoes successive phase transitions at 297, 291 and $263 \mathrm{~K}$ (Sugiyama et al., 1980). The room temperature phase (phase I) crystallizes in the orthorhombic space group Pmcm with Z = 4 (Morosin \& Lingafelter, 1961; Clay et al., 1975). Three low-temperature phases, named phases II, III and IV in the order of decreasing temperature, show incommensurate, ferroelastic commensurate monoclinic and monoclinic structures, respectively (Sugiyama et al., 1980; Gesi \& Iizumi, 1980). We allowed $\left[\left(\mathrm{CH}_{3}\right)_{4} \mathrm{~N}\right] \mathrm{Cl}, \mathrm{CuCl}_{2}$ and thioacetamide to react in ethanol. The expected mixed ligand complex was not crystallized but instead the title compound was obtained accidentally. The crystal structure of phase IV of the title compound was determined at $120 \mathrm{~K}$ and is reported herein.
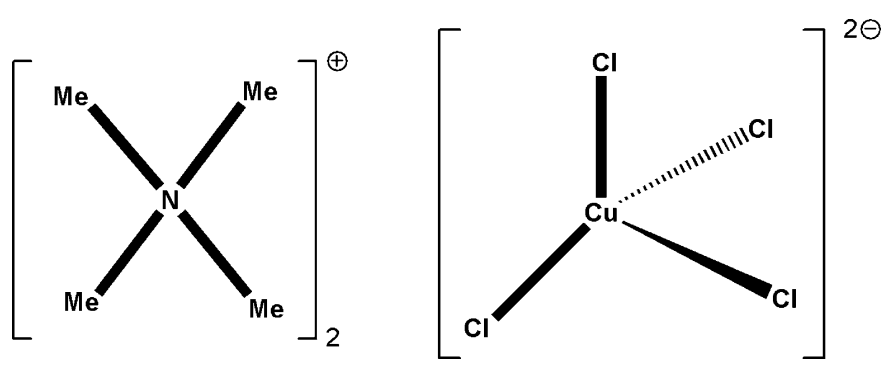

\section{Structural commentary}

The asymmetric unit of the title compound consists of a discrete $\left[\mathrm{CuCl}_{4}\right]^{2-}$ anion and two crystallographically tetramethylammonium cations (Fig. 1). In the anion, the four $\mathrm{Cl}$ atoms are inequivalent with $\mathrm{Cu}-\mathrm{Cl}$ distances ranging from $2.2313(15)$ to $2.2538(16) \AA$. The $\mathrm{Cl}-\mathrm{Cu}-\mathrm{Cl}$ angles vary from $98.44(7)$ to $133.69(7)^{\circ}$, indicating a distorted tetrahedral 

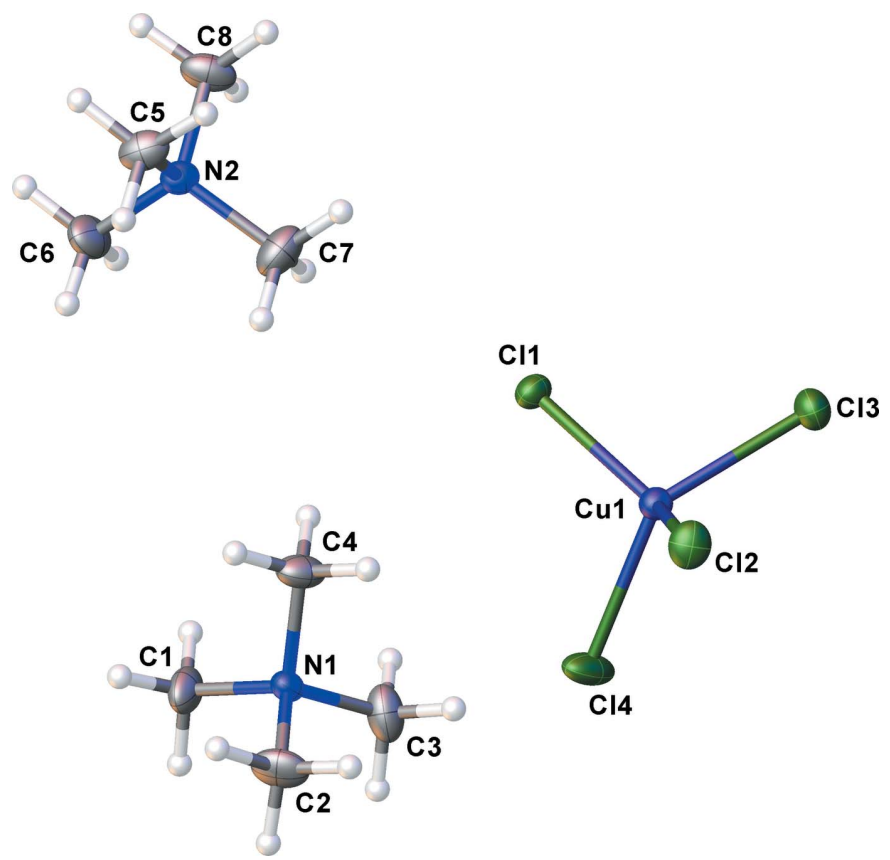

Figure 1

The asymmetric unit of the title compound showing the atom-labeling scheme. Displacement ellipsoids are drawn at the $50 \%$ probability level and $\mathrm{H}$ atoms are depicted as spheres of an arbitrary radius.

geometry around the $\mathrm{Cu}^{\mathrm{II}}$ atom. Using Houser's $\tau_{4}$ metric $\left[\tau_{4}=360-(\alpha+\beta) / 141\right]$, where $\alpha$ and $\beta$ are the largest angles about the metal atom (Yang et al., 2007), we obtain a value of 0.658 for phase IV and 0.792 for the orthorhombic phase I. This indicates a greater deviation from an ideal tetrahedron in phase IV compared with phase I, tending towards a 'see-saw' geometry.

\section{Supramolecular features}

In the crystal, the cations and the anions are linked via weak $\mathrm{C}-\mathrm{H}$... Cl hydrogen bonds (Table 1 and Fig. 2), forming a three-dimensional network.

\section{Database survey}

A substructure search for compounds that incorporate a tetramethylammonium ion and a copper tetrachloride species reveals thirteen structures (CSD November 2016; Groom et al., 2016). Of these, three are structures of $\left(\mathrm{Me}_{4} \mathrm{~N}\right)_{2}\left[\mathrm{CuCl}_{4}\right]$ with a discrete $\left[\mathrm{CuCl}_{4}\right]^{2-}$ anion (Morosin \& Lingafelter, 1961; Clay et al., 1975; Hlel et al., 2008).

\section{Synthesis and crystallization}

On mixing $\left[\left(\mathrm{CH}_{3}\right)_{4} \mathrm{~N}\right] \mathrm{Cl}(0.465 \mathrm{~g}, 4.2 \mathrm{mmol})$ in ethanol $(10 \mathrm{ml})$ with $\mathrm{CuCl}_{2} \cdot 2 \mathrm{H}_{2} \mathrm{O}(0.365 \mathrm{~g}, 2.1 \mathrm{mmol})$ in ethanol $(10 \mathrm{ml})$ and thioacetamide $(0.160 \mathrm{~g}, 2.1 \mathrm{mmol})$ in ethanol $(10 \mathrm{ml})$, a clear solution is obtained. Slow evaporation at room temperature $(301 \mathrm{~K})$ yielded pale-green crystals of $\left[\left(\mathrm{CH}_{3}\right)_{4} \mathrm{~N}\right]_{2}\left[\mathrm{CuCl}_{4}\right]$ suitable for X-ray determination.
Table 1

Hydrogen-bond geometry $\left(\AA{ }^{\circ}\right)$.

\begin{tabular}{lllll}
\hline$D-\mathrm{H} \cdots A$ & $D-\mathrm{H}$ & $\mathrm{H} \cdots A$ & $D \cdots A$ & $D-\mathrm{H} \cdots A$ \\
\hline $\mathrm{C} 1-\mathrm{H} 1 C \cdots \mathrm{Cl} 2^{\mathrm{i}}$ & 0.98 & 2.69 & $3.585(7)$ & 153 \\
$\mathrm{C} 2-\mathrm{H} 2 A \cdots \mathrm{Cl} 1^{\mathrm{ii}}$ & 0.98 & 2.79 & $3.675(7)$ & 151 \\
$\mathrm{C} 2-\mathrm{H} 2 A \cdots \mathrm{Cl} 3^{\mathrm{ii}}$ & 0.98 & 2.80 & $3.555(7)$ & 134 \\
$\mathrm{C} 2-\mathrm{H} 2 B \cdots \mathrm{Cl} 1^{\mathrm{i}}$ & 0.98 & 2.79 & $3.674(7)$ & 150 \\
$\mathrm{C} 3-\mathrm{H} 3 B \cdots \mathrm{Cl} 4$ & 0.98 & 2.74 & $3.670(7)$ & 159 \\
$\mathrm{C} 4-\mathrm{H} 4 A \cdots \mathrm{Cl} 3^{\mathrm{iii}}$ & 0.98 & 2.59 & $3.555(7)$ & 166 \\
$\mathrm{C} 5-\mathrm{H} 5 A \cdots \mathrm{Cl} 2^{\mathrm{iv}}$ & 0.98 & 2.68 & $3.635(7)$ & 165 \\
$\mathrm{C} 5-\mathrm{H} 5 B \cdots \mathrm{Cl} 3^{\mathrm{v}}$ & 0.98 & 2.81 & $3.587(6)$ & 137 \\
$\mathrm{C} 5-\mathrm{H} 5 C \cdots \mathrm{Cl} 4^{\mathrm{i}}$ & 0.98 & 2.63 & $3.610(6)$ & 173 \\
$\mathrm{C} 8-\mathrm{H} 8 B \cdots \mathrm{Cl} 1^{\mathrm{iii}}$ & 0.98 & 2.76 & $3.650(6)$ & 151 \\
$\mathrm{C} 8-\mathrm{H} 8 C \cdots \mathrm{Cl} 2^{\mathrm{v}}$ & 0.98 & 2.82 & $3.763(7)$ & 162 \\
\hline
\end{tabular}

Symmetry codes: (i) $-x+1,-y+1,-z+1$; (ii) $x-1, y, z$; (iii) $-x+\frac{3}{2}, y-\frac{1}{2},-z+\frac{3}{2}$; (iv) $x, y-1, z ;(\mathrm{v})-x+2,-y+1,-z+1$.

\section{Refinement}

Crystal data, data collection and structure refinement details are summarized in Table 2. $\mathrm{H}$ atoms were included in idealized geometries and allowed to rotate to minimize their electrondensity contribution with $\mathrm{C}-\mathrm{H}=0.98 \AA$ and $U_{\text {iso }}(\mathrm{H})=$ $1.5 U_{\text {eq }}(\mathrm{C})$. The crystal used was found to be twinned through a $180^{\circ}$ rotation about the reciprocal $a$ axis with a twin component ratio of 0.76:0.24 (matrix: [1.000 $-0.003 \quad 0.0040 .001$ $-1.000-0.003-0.0930 .005-1.000])$. The diffraction data were integrated routinely applying this matrix and were scaled for absorption effects using TWINABS (Krause et al., 2015). In the final model, incorporation of the twinned data did not

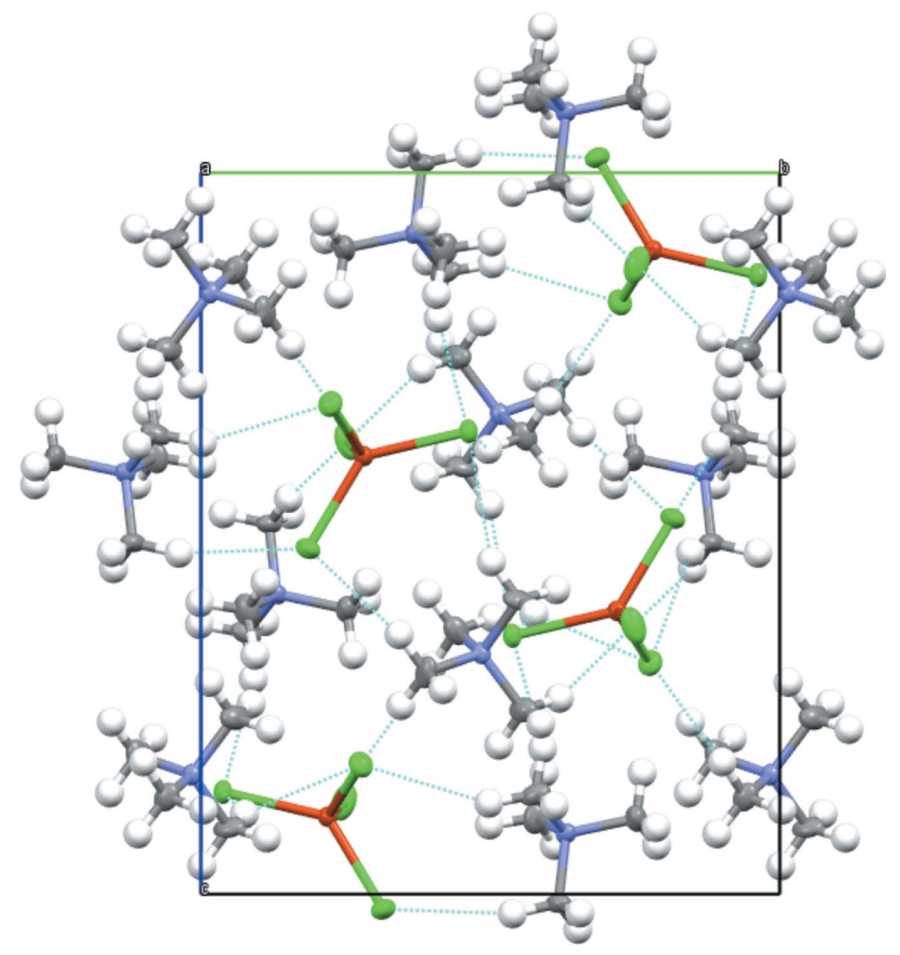

Figure 2

A packing diagram of the title compound, viewed along the $a$ axis, showing the $\mathrm{C}-\mathrm{H} \cdots \mathrm{Cl}$ hydrogen bonds (blue dashed lines). 
significantly alter the model, thus the final model was refined using the majority component data.

\section{Acknowledgements}

The authors acknowledge the Cheikh Anta Diop University of Dakar (Senegal) and the University of Notre Dame (USA) for equipment support.

\section{References}

Bruker (2015). APEX3 and SAINT. Bruker AXS Inc., Madison, Wisconsin, USA.

Clay, R., Murray-Rust, J. \& Murray-Rust, P. (1975). Acta Cryst. B31, 289-290.

Gesi, K. \& Iizumi, M. (1980). J. Phys. Soc. Jpn, 48, 1775-1776.

Groom, C. R., Bruno, I. J., Lightfoot, M. P. \& Ward, S. C. (2016). Acta Cryst. B72, 171-179.

Hlel, F., Ben Rhaeim, A. \& Guidara, K. (2008). Zh. Neorg. Khim. 53, 785-793.

Krause, L., Herbst-Irmer, R., Sheldrick, G. M. \& Stalke, D. (2015). J. Appl. Cryst. 48, 3-10.

Morosin, B. \& Lingafelter, E. C. (1961). J. Phys. Chem. 65, 50-51.

Sheldrick, G. M. (2008). Acta Cryst. A64, 112-122.

Sheldrick, G. M. (2015a). Acta Cryst. A71, 3-8.

Sheldrick, G. M. (2015b). Acta Cryst. C71, 3-8.

Sugiyama, J., Wada, M., Sawada, A. \& Ishibashi, Y. (1980). J. Phys. Soc. Jpn, 49, 1405-1412.

Westrip, S. P. (2010). J. Appl. Cryst. 43, 920-925.

Yang, L., Powell, D. R. \& Houser, R. P. (2007). Dalton Trans. pp. 955964.
Table 2

Experimental details.

\begin{tabular}{|c|c|}
\hline \multicolumn{2}{|l|}{ Crystal data } \\
\hline Chemical formula & $\left(\mathrm{C}_{4} \mathrm{H}_{12} \mathrm{~N}\right)_{2}\left[\mathrm{CuCl}_{4}\right]$ \\
\hline$M_{\mathrm{r}}$ & 353.63 \\
\hline Crystal system, space group & Monoclinic, $P 2_{1} / n$ \\
\hline Temperature $(\mathrm{K})$ & 120 \\
\hline$a, b, c(\AA)$ & $8.9901(5), 12.0059(7), 14.9570(9)$ \\
\hline$\beta\left(^{\circ}\right)$ & $91.719(3)$ \\
\hline$V\left(\AA^{3}\right)$ & $1613.65(16)$ \\
\hline$Z$ & 4 \\
\hline Radiation type & Мо $K \alpha$ \\
\hline$\mu\left(\mathrm{mm}^{-1}\right)$ & 1.99 \\
\hline Crystal size $(\mathrm{mm})$ & $0.15 \times 0.12 \times 0.11$ \\
\hline \multicolumn{2}{|l|}{ Data collection } \\
\hline Diffractometer & Bruker APEXII \\
\hline Absorption correction & $\begin{array}{l}\text { Multi-scan (TWINABS; Krause et } \\
\quad \text { al., 2015) }\end{array}$ \\
\hline$T_{\min }, T_{\max }$ & $0.659,0.746$ \\
\hline $\begin{array}{l}\text { No. of measured, independent and } \\
\text { observed }[I>2 \sigma(I)] \text { reflections }\end{array}$ & $7842,4019,2862$ \\
\hline$R_{\text {int }}$ & 0.057 \\
\hline$(\sin \theta / \lambda)_{\max }\left(\AA^{-1}\right)$ & 0.669 \\
\hline \multicolumn{2}{|l|}{ Refinement } \\
\hline$R\left[F^{2}>2 \sigma\left(F^{2}\right)\right], w R\left(F^{2}\right), S$ & $0.066,0.141,1.12$ \\
\hline No. of reflections & 4019 \\
\hline No. of parameters & 144 \\
\hline $\mathrm{H}$-atom treatment & $\mathrm{H}$-atom parameters constrained \\
\hline$\Delta \rho_{\max }, \Delta \rho_{\min }\left(\mathrm{e} \AA^{-3}\right)$ & $1.16,-1.03$ \\
\hline
\end{tabular}

Computer programs: APEX3 and SAINT (Bruker, 2015), SHELXT (Sheldrick, 2015a), SHELXL2014 (Sheldrick, 2015b), XP in SHELXTL (Sheldrick, 2008) and publCIF (Westrip, 2010). 


\section{supporting information}

Acta Cryst. (2017). E73, 358-360 [https://doi.org/10.1107/S2056989017002146]

\section{Crystal structure of the monoclinic phase (phase IV) of bis(tetramethyl- ammonium) tetrachloridocuprate(II)}

\section{Gorgui Awa Seck, Libasse Diop and Allen G. Oliver}

\section{Computing details}

Data collection: APEX3 (Bruker, 2015); cell refinement: SAINT (Bruker, 2015); data reduction: SAINT (Bruker, 2015); program(s) used to solve structure: SHELXT (Sheldrick, 2015a); program(s) used to refine structure: SHELXL2014 (Sheldrick, 2015b); molecular graphics: XP in SHELXTL (Sheldrick, 2008); software used to prepare material for publication: publCIF (Westrip, 2010).

Bis(tetramethylammonium) tetrachloridocuprate(II)

\section{Crystal data}

$\left(\mathrm{C}_{4} \mathrm{H}_{12} \mathrm{~N}\right)_{2}\left[\mathrm{CuCl}_{4}\right]$

$M_{r}=353.63$

Monoclinic, $P 2_{1} / n$

$a=8.9901(5) \AA$

$b=12.0059(7) \AA$

$c=14.9570(9) \AA$

$\beta=91.719(3)^{\circ}$

$V=1613.65(16) \AA^{3}$

$Z=4$

\section{Data collection}

Bruker APEXII

diffractometer

Radiation source: fine-focus sealed tube

Graphite monochromator

Detector resolution: 8.33 pixels $\mathrm{mm}^{-1}$

combination of $\omega$ and $\varphi$-scans

Absorption correction: multi-scan

(TWINABS; Krause et al., 2015)

$T_{\min }=0.659, T_{\max }=0.746$

\section{Refinement}

Refinement on $F^{2}$

Least-squares matrix: full

$R\left[F^{2}>2 \sigma\left(F^{2}\right)\right]=0.066$

$w R\left(F^{2}\right)=0.141$

$S=1.12$

4019 reflections

144 parameters

0 restraints
$F(000)=732$

$D_{\mathrm{x}}=1.456 \mathrm{Mg} \mathrm{m}^{-3}$

Mo $K \alpha$ radiation, $\lambda=0.71073 \AA$

Cell parameters from 6598 reflections

$\theta=2.6-24.7^{\circ}$

$\mu=1.99 \mathrm{~mm}^{-1}$

$T=120 \mathrm{~K}$

Block, pale green

$0.15 \times 0.12 \times 0.11 \mathrm{~mm}$

7842 measured reflections

4019 independent reflections

2862 reflections with $I>2 \sigma(I)$

$R_{\text {int }}=0.057$

$\theta_{\max }=28.4^{\circ}, \theta_{\min }=2.2^{\circ}$

$h=-12 \rightarrow 12$

$k=-16 \rightarrow 16$

$l=0 \rightarrow 19$

Primary atom site location: real-space vector search

Secondary atom site location: difference Fourier map

Hydrogen site location: inferred from neighbouring sites

$\mathrm{H}$-atom parameters constrained 
$w=1 /\left[\sigma^{2}\left(F_{\mathrm{o}}^{2}\right)+15.8242 P\right]$

where $P=\left(F_{\mathrm{o}}{ }^{2}+2 F_{\mathrm{c}}{ }^{2}\right) / 3$

$(\Delta / \sigma)_{\max }<0.001$

$$
\Delta \rho_{\max }=1.16 \text { e } \AA^{-3}
$$

\section{Special details}

Geometry. All esds (except the esd in the dihedral angle between two 1.s. planes) are estimated using the full covariance matrix. The cell esds are taken into account individually in the estimation of esds in distances, angles and torsion angles; correlations between esds in cell parameters are only used when they are defined by crystal symmetry. An approximate (isotropic) treatment of cell esds is used for estimating esds involving l.s. planes.

Fractional atomic coordinates and isotropic or equivalent isotropic displacement parameters $\left(\hat{A}^{2}\right)$

\begin{tabular}{|c|c|c|c|c|}
\hline & $x$ & $y$ & $z$ & $U_{\text {iso }} * / U_{\text {eq }}$ \\
\hline $\mathrm{Cu} 1$ & $0.77037(7)$ & $0.71890(6)$ & $0.60734(5)$ & $0.01762(17)$ \\
\hline Cl1 & $0.78370(15)$ & $0.53786(11)$ & $0.64164(10)$ & $0.0211(3)$ \\
\hline $\mathrm{Cl} 2$ & $0.79189(17)$ & $0.81507(13)$ & $0.47896(10)$ & $0.0275(3)$ \\
\hline $\mathrm{Cl} 3$ & $0.97801(15)$ & $0.77628(13)$ & $0.67958(10)$ & $0.0267(3)$ \\
\hline $\mathrm{Cl} 4$ & $0.52836(16)$ & $0.74708(14)$ & 0.62755 (13) & $0.0363(4)$ \\
\hline N1 & $0.2548(5)$ & $0.4839(4)$ & $0.6659(3)$ & $0.0183(10)$ \\
\hline $\mathrm{C} 1$ & $0.1601(7)$ & $0.3893(5)$ & $0.6925(5)$ & $0.0322(15)$ \\
\hline $\mathrm{H} 1 \mathrm{~A}$ & 0.0671 & 0.4177 & 0.7162 & $0.048^{*}$ \\
\hline H1B & 0.2128 & 0.3452 & 0.7386 & $0.048^{*}$ \\
\hline $\mathrm{H} 1 \mathrm{C}$ & 0.1378 & 0.3422 & 0.6403 & $0.048^{*}$ \\
\hline $\mathrm{C} 2$ & $0.1815(7)$ & $0.5491(6)$ & $0.5923(4)$ & $0.0328(15)$ \\
\hline $\mathrm{H} 2 \mathrm{~A}$ & 0.0840 & 0.5750 & 0.6113 & $0.049^{*}$ \\
\hline $\mathrm{H} 2 \mathrm{~B}$ & 0.1685 & 0.5017 & 0.5392 & $0.049^{*}$ \\
\hline $\mathrm{H} 2 \mathrm{C}$ & 0.2436 & 0.6133 & 0.5779 & $0.049^{*}$ \\
\hline $\mathrm{C} 3$ & $0.2801(7)$ & $0.5574(6)$ & $0.7446(5)$ & $0.0344(16)$ \\
\hline $\mathrm{H} 3 \mathrm{~A}$ & 0.1846 & 0.5868 & 0.7639 & $0.052 *$ \\
\hline H3B & 0.3451 & 0.6192 & 0.7284 & $0.052 *$ \\
\hline $\mathrm{H} 3 \mathrm{C}$ & 0.3273 & 0.5146 & 0.7935 & $0.052 *$ \\
\hline $\mathrm{C} 4$ & $0.4008(6)$ & $0.4411(6)$ & $0.6347(5)$ & $0.0296(14)$ \\
\hline $\mathrm{H} 4 \mathrm{~A}$ & 0.4516 & 0.4002 & 0.6834 & $0.044^{*}$ \\
\hline $\mathrm{H} 4 \mathrm{~B}$ & 0.4629 & 0.5038 & 0.6167 & $0.044^{*}$ \\
\hline $\mathrm{H} 4 \mathrm{C}$ & 0.3836 & 0.3912 & 0.5836 & $0.044^{*}$ \\
\hline $\mathrm{N} 2$ & $0.7505(5)$ & $0.1309(4)$ & $0.5840(3)$ & $0.0198(10)$ \\
\hline $\mathrm{C} 5$ & $0.7809(7)$ & $0.1175(6)$ & $0.4871(4)$ & $0.0279(14)$ \\
\hline $\mathrm{H} 5 \mathrm{~A}$ & 0.7882 & 0.0381 & 0.4727 & $0.042 *$ \\
\hline H5B & 0.8748 & 0.1545 & 0.4738 & $0.042 *$ \\
\hline $\mathrm{H} 5 \mathrm{C}$ & 0.6998 & 0.1511 & 0.4512 & $0.042 *$ \\
\hline C6 & $0.6058(7)$ & $0.0788(6)$ & $0.6059(5)$ & $0.0334(16)$ \\
\hline H6A & 0.6112 & -0.0018 & 0.5957 & $0.050^{*}$ \\
\hline H6B & 0.5264 & 0.1106 & 0.5675 & $0.050^{*}$ \\
\hline $\mathrm{H} 6 \mathrm{C}$ & 0.5846 & 0.0931 & 0.6687 & $0.050^{*}$ \\
\hline $\mathrm{C} 7$ & $0.7444(8)$ & $0.2520(5)$ & $0.6048(5)$ & $0.0347(16)$ \\
\hline H7A & 0.7225 & 0.2622 & 0.6681 & $0.052^{*}$ \\
\hline H7B & 0.6661 & 0.2872 & 0.5676 & $0.052^{*}$ \\
\hline $\mathrm{H} 7 \mathrm{C}$ & 0.8405 & 0.2863 & 0.5925 & $0.052 *$ \\
\hline $\mathrm{C} 8$ & $0.8722(7)$ & $0.0776(6)$ & $0.6381(4)$ & $0.0304(15)$ \\
\hline
\end{tabular}


supporting information

$\begin{array}{lllll}\text { H8A } & 0.8733 & -0.0026 & 0.6260 & 0.046^{*} \\ \text { H8B } & 0.8558 & 0.0901 & 0.7018 & 0.046^{*} \\ \text { H8C } & 0.9678 & 0.1102 & 0.6222 & 0.046^{*}\end{array}$

Atomic displacement parameters $\left(\AA^{2}\right)$

\begin{tabular}{lllllll}
\hline & $U^{11}$ & $U^{22}$ & $U^{33}$ & $U^{12}$ & $U^{13}$ & $U^{23}$ \\
\hline $\mathrm{Cu} 1$ & $0.0169(3)$ & $0.0172(3)$ & $0.0188(3)$ & $0.0000(3)$ & $0.0018(2)$ & $0.0003(3)$ \\
$\mathrm{C} 11$ & $0.0216(7)$ & $0.0157(6)$ & $0.0261(7)$ & $0.0009(5)$ & $0.0031(5)$ & $0.0006(5)$ \\
$\mathrm{Cl} 2$ & $0.0355(8)$ & $0.0277(8)$ & $0.0195(7)$ & $-0.0040(6)$ & $0.0024(6)$ & $0.0043(6)$ \\
$\mathrm{Cl} 3$ & $0.0227(7)$ & $0.0268(8)$ & $0.0304(8)$ & $-0.0034(6)$ & $-0.0019(6)$ & $-0.0028(6)$ \\
$\mathrm{C} 14$ & $0.0169(7)$ & $0.0303(8)$ & $0.0621(12)$ & $0.0049(6)$ & $0.0097(7)$ & $0.0129(8)$ \\
$\mathrm{N} 1$ & $0.014(2)$ & $0.018(2)$ & $0.022(3)$ & $0.0001(18)$ & $-0.0003(18)$ & $0.0018(19)$ \\
$\mathrm{C} 1$ & $0.028(3)$ & $0.022(3)$ & $0.047(4)$ & $-0.010(3)$ & $0.012(3)$ & $-0.005(3)$ \\
$\mathrm{C} 2$ & $0.030(3)$ & $0.048(4)$ & $0.020(3)$ & $0.013(3)$ & $-0.004(3)$ & $0.007(3)$ \\
$\mathrm{C} 3$ & $0.038(4)$ & $0.037(4)$ & $0.028(4)$ & $-0.016(3)$ & $-0.001(3)$ & $-0.001(3)$ \\
$\mathrm{C} 4$ & $0.020(3)$ & $0.030(4)$ & $0.038(4)$ & $0.006(3)$ & $0.008(3)$ & $0.007(3)$ \\
$\mathrm{N} 2$ & $0.020(2)$ & $0.020(2)$ & $0.019(3)$ & $0.0022(19)$ & $0.0043(19)$ & $0.0018(19)$ \\
$\mathrm{C} 5$ & $0.036(4)$ & $0.031(3)$ & $0.016(3)$ & $0.009(3)$ & $0.002(3)$ & $0.001(3)$ \\
$\mathrm{C} 6$ & $0.021(3)$ & $0.031(4)$ & $0.048(4)$ & $-0.006(3)$ & $0.007(3)$ & $0.005(3)$ \\
$\mathrm{C} 7$ & $0.051(4)$ & $0.022(3)$ & $0.032(4)$ & $0.001(3)$ & $0.009(3)$ & $-0.002(3)$ \\
$\mathrm{C} 8$ & $0.025(3)$ & $0.043(4)$ & $0.023(3)$ & $0.009(3)$ & $0.001(2)$ & $0.010(3)$ \\
& & & & & & \\
\hline
\end{tabular}

Geometric parameters $\left(\AA,{ }^{\circ}\right)$

\begin{tabular}{llll}
\hline $\mathrm{Cu} 1-\mathrm{Cl} 4$ & $2.2313(15)$ & $\mathrm{C} 4-\mathrm{H} 4 \mathrm{~B}$ & 0.9800 \\
$\mathrm{Cu} 1-\mathrm{Cl1}$ & $2.2357(15)$ & $\mathrm{C} 4-\mathrm{H} 4 \mathrm{C}$ & 0.9800 \\
$\mathrm{Cu} 1-\mathrm{C} 13$ & $2.2374(15)$ & $\mathrm{N} 2-\mathrm{C} 8$ & $1.486(7)$ \\
$\mathrm{Cu} 1-\mathrm{C} 12$ & $2.2538(16)$ & $\mathrm{N} 2-\mathrm{C} 7$ & $1.488(8)$ \\
$\mathrm{N} 1-\mathrm{C} 1$ & $1.481(7)$ & $\mathrm{N} 2-\mathrm{C} 6$ & $1.488(7)$ \\
$\mathrm{N} 1-\mathrm{C} 3$ & $1.483(8)$ & $\mathrm{N} 2-\mathrm{C} 5$ & $1.491(7)$ \\
$\mathrm{N} 1-\mathrm{C} 2$ & $1.488(7)$ & $\mathrm{C} 5-\mathrm{H} 5 \mathrm{~A}$ & 0.9800 \\
$\mathrm{~N} 1-\mathrm{C} 4$ & $1.498(7)$ & $\mathrm{C} 5-\mathrm{H} 5 \mathrm{~B}$ & 0.9800 \\
$\mathrm{C} 1-\mathrm{H} 1 \mathrm{~A}$ & 0.9800 & $\mathrm{C} 5-\mathrm{H} 5 \mathrm{C}$ & 0.9800 \\
$\mathrm{C} 1-\mathrm{H} 1 \mathrm{~B}$ & 0.9800 & $\mathrm{C} 6-\mathrm{H} 6 \mathrm{~A}$ & 0.9800 \\
$\mathrm{C} 1-\mathrm{H} 1 \mathrm{C}$ & 0.9800 & $\mathrm{C} 6-\mathrm{H} 6 \mathrm{~B}$ & 0.9800 \\
$\mathrm{C} 2-\mathrm{H} 2 \mathrm{~A}$ & 0.9800 & $\mathrm{C} 6-\mathrm{H} 6 \mathrm{C}$ & 0.9800 \\
$\mathrm{C} 2-\mathrm{H} 2 \mathrm{~B}$ & 0.9800 & $\mathrm{C} 7-\mathrm{H} 7 \mathrm{~A}$ & 0.9800 \\
$\mathrm{C} 2-\mathrm{H} 2 \mathrm{C}$ & 0.9800 & $\mathrm{C} 7-\mathrm{H} 7 \mathrm{~B}$ & 0.9800 \\
$\mathrm{C} 3-\mathrm{H} 3 \mathrm{~A}$ & 0.9800 & $\mathrm{C} 7-\mathrm{H} 7 \mathrm{C}$ & 0.9800 \\
$\mathrm{C} 3-\mathrm{H} 3 \mathrm{~B}$ & 0.9800 & $\mathrm{C} 8-\mathrm{H} 8 \mathrm{~A}$ & 0.9800 \\
$\mathrm{C} 3-\mathrm{H} 3 \mathrm{C}$ & 0.9800 & $\mathrm{C} 8-\mathrm{H} 8 \mathrm{~B}$ & 0.9800 \\
$\mathrm{C} 4-\mathrm{H} 4 \mathrm{~A}$ & 0.9800 & & 0.9800 \\
& & $\mathrm{~N} 1-\mathrm{C} 4-\mathrm{H} 4 \mathrm{C}$ & \\
$\mathrm{C} 14-\mathrm{Cu} 1-\mathrm{C} 11$ & $99.33(6)$ & $\mathrm{H} 4 \mathrm{~A}-\mathrm{C} 4-\mathrm{H} 4 \mathrm{C}$ & 109.5 \\
$\mathrm{C} 14-\mathrm{Cu} 1-\mathrm{Cl} 3$ & $133.69(7)$ & $\mathrm{C} 8-\mathrm{B} 2-\mathrm{C} 4-\mathrm{H} 4 \mathrm{C}$ & 109.5 \\
$\mathrm{C} 11-\mathrm{Cu} 1-\mathrm{Cl3}$ & $98.64(6)$ & & 109.5 \\
$\mathrm{C} 14-\mathrm{Cu} 1-\mathrm{Cl} 2$ & $98.44(7)$ & &
\end{tabular}




$\begin{array}{ll}\mathrm{C} 11-\mathrm{Cu} 1-\mathrm{Cl} 2 & 133.48(6) \\ \mathrm{C} 13-\mathrm{Cu} 1-\mathrm{Cl} 2 & 99.32(6) \\ \mathrm{C} 1-\mathrm{N} 1-\mathrm{C} 3 & 108.6(5) \\ \mathrm{C} 1-\mathrm{N} 1-\mathrm{C} 2 & 110.9(5) \\ \mathrm{C} 3-\mathrm{N} 1-\mathrm{C} 2 & 109.2(5) \\ \mathrm{C} 1-\mathrm{N} 1-\mathrm{C} 4 & 109.7(5) \\ \mathrm{C} 3-\mathrm{N} 1-\mathrm{C} 4 & 109.6(5) \\ \mathrm{C} 2-\mathrm{N} 1-\mathrm{C} 4 & 108.9(5) \\ \mathrm{N} 1-\mathrm{C} 1-\mathrm{H} 1 \mathrm{~A} & 109.5 \\ \mathrm{~N} 1-\mathrm{C} 1-\mathrm{H} 1 \mathrm{~B} & 109.5 \\ \mathrm{H} 1 \mathrm{~A}-\mathrm{C} 1-\mathrm{H} 1 \mathrm{~B} & 109.5 \\ \mathrm{~N} 1-\mathrm{C} 1-\mathrm{H} 1 \mathrm{C} & 109.5 \\ \mathrm{H} 1 \mathrm{~A}-\mathrm{C} 1-\mathrm{H} 1 \mathrm{C} & 109.5 \\ \mathrm{H} 1 \mathrm{~B}-\mathrm{C} 1-\mathrm{H} 1 \mathrm{C} & 109.5 \\ \mathrm{~N} 1-\mathrm{C} 2-\mathrm{H} 2 \mathrm{~A} & 109.5 \\ \mathrm{~N} 1-\mathrm{C} 2-\mathrm{H} 2 \mathrm{~B} & 109.5 \\ \mathrm{H} 2 \mathrm{~A}-\mathrm{C} 2-\mathrm{H} 2 \mathrm{~B} & 109.5 \\ \mathrm{~N} 1-\mathrm{C} 2-\mathrm{H} 2 \mathrm{C} & 109.5 \\ \mathrm{H} 2 \mathrm{~A}-\mathrm{C} 2-\mathrm{H} 2 \mathrm{C} & 109.5 \\ \mathrm{H} 2 \mathrm{~B}-\mathrm{C} 2-\mathrm{H} 2 \mathrm{C} & 109.5 \\ \mathrm{~N} 1-\mathrm{C} 3-\mathrm{H} 3 \mathrm{~A} & 109.5 \\ \mathrm{~N} 1-\mathrm{C} 3-\mathrm{H} 3 \mathrm{~B} & 109.5 \\ \mathrm{H} 3 \mathrm{~A}-\mathrm{C} 3-\mathrm{H} 3 \mathrm{~B} & 109.5 \\ \mathrm{~N} 1-\mathrm{C} 3-\mathrm{H} 3 \mathrm{C} & 109.5 \\ \mathrm{H} 3 \mathrm{~A}-\mathrm{C} 3-\mathrm{H} 3 \mathrm{C} & 109.5 \\ \mathrm{H} 3 \mathrm{~B}-\mathrm{C} 3-\mathrm{H} 3 \mathrm{C} & 109.5 \\ \mathrm{~N} 1-\mathrm{C} 4-\mathrm{H} 4 \mathrm{~A} & 109.5 \\ \mathrm{~N} 1-\mathrm{C} 4-\mathrm{H} 4 \mathrm{~B} & 109.5 \\ \mathrm{H} 4 \mathrm{~A}-\mathrm{C} 4-\mathrm{H} 4 \mathrm{~B} & 109.5 \\ & \end{array}$

$\begin{array}{ll}\text { C8-N2-C6 } & 109.5(5) \\ \text { C7-N2-C6 } & 109.1(5) \\ \text { C8-N2-C5 } & 109.2(4) \\ \text { C7-N2-C5 } & 108.5(5) \\ \text { C6-N2-C5 } & 110.8(5) \\ \text { N2-C5-H5A } & 109.5 \\ \text { N2-C5-H5B } & 109.5 \\ \text { H5A-C5-H5B } & 109.5 \\ \text { N2-C5-H5C } & 109.5 \\ \text { H5A-C5-H5C } & 109.5 \\ \text { H5B-C5-H5C } & 109.5 \\ \text { N2-C6-H6A } & 109.5 \\ \text { N2-C6-H6B } & 109.5 \\ \text { H6A-C6-H6B } & 109.5 \\ \text { N2-C6-H6C } & 109.5 \\ \text { H6A-C6-H6C } & 109.5 \\ \text { H6B-C6-H6C } & 109.5 \\ \text { N2-C7-H7A } & 109.5 \\ \text { N2-C7-H7B } & 109.5 \\ \text { H7A-C7-H7B } & 109.5 \\ \text { N2-C7-H7C } & 109.5 \\ \text { H7A-C7-H7C } & 109.5 \\ \text { H7B-C7-H7C } & 109.5 \\ \text { N2-C8-H8A } & 109.5 \\ \text { N2-C8-H8B } & 109.5 \\ \text { H8A-C8-H8B } & 109.5 \\ \text { N2-C8-H8C } & 109.5 \\ \text { H8A-C8-H8C } & 109.5 \\ \text { H8B-C8-H8C } & 109.5 \\ & \end{array}$

Hydrogen-bond geometry $\left(\AA,{ }^{\circ}\right)$

\begin{tabular}{lllll}
\hline$D-\mathrm{H} \cdots A$ & $D-\mathrm{H}$ & $\mathrm{H} \cdots A$ & $D \cdots A$ & $D-\mathrm{H} \cdots A$ \\
\hline $\mathrm{C} 1-\mathrm{H} 1 C \cdots \mathrm{Cl} 2^{\mathrm{i}}$ & 0.98 & 2.69 & $3.585(7)$ & 153 \\
$\mathrm{C} 2-\mathrm{H} 2 A \cdots \mathrm{Cl} 1^{\mathrm{ii}}$ & 0.98 & 2.79 & $3.675(7)$ & 151 \\
$\mathrm{C} 2-\mathrm{H} 2 A \cdots \mathrm{Cl} 3^{\mathrm{ii}}$ & 0.98 & 2.80 & $3.555(7)$ & 134 \\
$\mathrm{C} 2-\mathrm{H} 2 B \cdots \mathrm{Cl} 1^{\mathrm{i}}$ & 0.98 & 2.79 & $3.674(7)$ & 150 \\
$\mathrm{C} 3-\mathrm{H} 3 B \cdots \mathrm{Cl} 4$ & 0.98 & 2.74 & $3.670(7)$ & 159 \\
$\mathrm{C} 4-\mathrm{H} 4 A \cdots \mathrm{Cl} 3^{\mathrm{iii}}$ & 0.98 & 2.59 & $3.555(7)$ & 166 \\
$\mathrm{C} 5-\mathrm{H} 5 A \cdots \mathrm{Cl} 2^{\mathrm{iv}}$ & 0.98 & 2.68 & $3.635(7)$ & 165 \\
$\mathrm{C} 5-\mathrm{H} 5 B \cdots \mathrm{Cl} 3^{\mathrm{v}}$ & 0.98 & 2.81 & $3.587(6)$ & 137 \\
$\mathrm{C} 5-\mathrm{H} 5 C \cdots \mathrm{Cl} 4^{\mathrm{i}}$ & 0.98 & 2.63 & $3.610(6)$ & 173 \\
$\mathrm{C} 8-\mathrm{H} 8 B \cdots \mathrm{Cl} 1^{\mathrm{iii}}$ & 0.98 & 2.76 & $3.650(6)$ & 151 \\
$\mathrm{C} 8-\mathrm{H} 8 C \cdots \mathrm{Cl} 2^{\mathrm{v}}$ & 0.98 & 2.82 & $3.763(7)$ & 162 \\
\hline
\end{tabular}

Symmetry codes: (i) $-x+1,-y+1,-z+1$; (ii) $x-1, y, z$; (iii) $-x+3 / 2, y-1 / 2,-z+3 / 2$; (iv) $x, y-1, z$; (v) $-x+2,-y+1,-z+1$. 